\title{
REVIEW
}

\section{New frontiers and emerging applications of 3D printing in ENT surgery: a systematic review of the literature}

\author{
Nuove frontiere e applicazioni emergenti della stampa 3D in ORL: \\ revisione sistematica della letteratura \\ P. Canzi ${ }^{1}$, M. Magnetto ${ }^{1}$, S. Marconi², P. Morbini ${ }^{3}$, S. Mauramati ${ }^{1}$, F. Aprile ${ }^{1}$, I. Avato ${ }^{1,4}$, \\ F. Auricchio², M. Benazzo \\ ${ }^{1}$ Department of Otorhinolaryngology, University of Pavia, Foundation IRCCS Policlinico "San Matteo", Pavia, Italy; \\ ${ }^{2}$ Department of Civil Engineering and Architecture, University of Pavia, Italy; ${ }^{3}$ Department of Pathology, University of \\ Pavia, Foundation IRCCS Policlinico S. Matteo, Pavia, Italy; ${ }^{4}$ PhD in Experimental Medicine, University of Pavia, Italy
}

\begin{abstract}
SUMMARY
3D printing systems have revolutionised prototyping in the industrial field by lowering production time from days to hours and costs from thousands to just a few dollars. Today, 3D printers are no more confined to prototyping, but are increasingly employed in medical disciplines with fascinating results, even in many aspects of otorhinolaryngology. All publications on ENT surgery, sourced through updated electronic databases (PubMed, MEDLINE, EMBASE) and published up to March 2017, were examined according to PRISMA guidelines. Overall, 121 studies fulfilled specific inclusion criteria and were included in our systematic review. Studies were classified according to the specific field of application (otologic, rhinologic, head and neck) and area of interest (surgical and preclinical education, customised surgical planning, tissue engineering and implantable prosthesis). Technological aspects, clinical implications and limits of 3D printing processes are discussed focusing on current benefits and future perspectives.
\end{abstract}

KEY WORDS: 3D printing $\bullet$ Additive manufacturing $\bullet$ Rapid prototyping $\bullet$ Otorhinolaryngology $\bullet$ ENT $\bullet$ Systematic review

\section{RIASSUNTO}

Le tecnologie di stampa $3 D$ hanno rivoluzionato la realizzazione di prototipi in ambito industriale, riducendo $i$ tempi ed $i$ costi di produzione rispettivamente da giorni ad ore, da migliaia a pochi dollari. Ad oggi, $i$ sistemi di stampa $3 D$ non sono solamente confinati alla creazione di prototipi, ma hanno trovato un crescente impiego in medicina con risultati affascinanti anche nel campo dell'Otorinolaringoiatria. Applicando le linee guida "PRISMA", abbiamo svolto una revisione sistematica della letteratura al fine di esaminare tutti gli articoli inerenti l'Otorinolaringoiatria, che sono stati riportati sui database elettronici (PubMed, MEDLINE, EMBASE) aggiornati fino a Marzo 2017. Complessivamente, 121 studi scientifici hanno soddisfatto specifici criteri di inclusione e sono stati sottoposti alla nostra revisione sistematica. Le pubblicazioni sono state classificate in relazione al campo di applicazione specifico (otologico, rinologico, testacollo) e all'area di interesse (formazione chirurgica e preclinica, pianificazione prechirurgica personalizzata, ingegneria tissutale e protesi impiantabile). Gli aspetti tecnologici, le implicazioni cliniche ed i limiti delle tecnologie di stampa $3 D$ sono stati ampiamente discussi in riferimento agli effettivi vantaggi attuali ed alle prospettive future.

PAROLE CHIAVE: Stampa 3D・Prototipizzazione rapida $\bullet$ Produzione additiva $\bullet$ Otorinolaringoiatria $\bullet$ ORL $\bullet$ Revisione sistematica

Acta Otorhinolaryngol Ital 2018;38:286-303

\section{Introduction}

Around 1450, Gutenberg developed a printing system that became a stepping-stone in the timeline of communication technology, and considered as one of the most influential events in the sharing of scientific and medical knowledge.
Since its first introduction in the early 1980s, 3D printing (3DP) technology has rapidly caught the interest of the industry, healthcare and media with an overall business of $\$ 700$ million ${ }^{1-4}$. The nature of all 3D printers is the creation of a wide range of 3D objects obtained from digital data of easy management and available in open-access digital databas- 
es, allowing a unique opportunity for information exchange (e.g. 3dprint.nih.gov). Almost anything can be produced by 3DP systems: fuel injectors for rockets, jewels and hearing aid shells ${ }^{56}$. One of the most fascinating aspects of this technology concerns the employment of imaging studies. Today, radiology plays a pivotal role in diagnostic and therapeutic decision making. However, scans are still displayed on flat screens, resulting in a 2D representation of reality. Surgeons' experience the difficult task of figuring out a three-dimensional image on a daily basis, by analysing CT or MRI-slices in separate two-dimensional axial, coronal and sagittal projections ${ }^{7}$. 3DP systems allow to restore the third dimension that is lacking during visualisation of radiological image data. Along with the production of anatomical models addressed to customised surgical planning, medical teaching and surgical training, research in 3DP has explored the pioneering world of biologic tissue engineering, patient-specific implantation and ultimately of personalised pharmacoprinting. The increasing impact of 3DP processes in the scientific literature has recently involved many aspects of otorhinolaryngology, often followed by great expectations regarding patient care. Up to now, what are the applications of 3DP technologies in ENT surgery? Does this tool provide any substantial benefits in the ENT field? And what about future perspectives? The present work aims to answer these questions by carrying out a systematic review of the literature on the topic, a task that, to the best of our knowledge, has not undertaken previously.

\section{The technology of 3DP systems}

3DP is a subset of additive manufacturing (AM) or rapid prototyping in which objects are achieved by gradually layering material, rather than by subtraction from the raw material as is in the case of conventional technologies ${ }^{8}$. The main advantages of AM are its flexibility, precision and relative quickness in creating customised physical structures of almost any complex shape in a myriad of materials. Historically, 3DP processes were employed by the manufacturing industry to rapidly produce a representation of a system or a part before final release or commercialisation ${ }^{9}$. The 3DP was first conceived by C. Hull in 1986 as an "apparatus for production of three-dimensional objects by stereolithography" ${ }^{3}$. During the same year, he also developed the "Standard Triangulation Language" (.STL) file format, which makes it possible to deconstruct the surface of a three-dimensional object in a series of triangles. The .STL file can be obtained from a 3D "Computer-Aided Design" (CAD) software, a medical scan data (e.g. CT scan, MRI), or from existing objects by using point or laser scanners. This virtual model is subsequently sliced into thin 2D layers, which are then sent to the $3 \mathrm{D}$ printer. 3DP methodologies differ from one another in the way that materials are deployed and cured ${ }^{8}$. Recently, the ASTM International Committee F42 classified 3DP technologies in 7 different working process categories ${ }^{10}$ (Fig. 1).

I. Vat photopolymerisation: in this technique a container gets filled with photopolymeric resin. This resin is then hardened by an UV light source.

II. Material jetting: this process resembles inkjet paper printing, since the material is dropped through small diameter nozzles. In this case, the base material is a photopolymeric resin subsequently hardened by a UV lamp.

III. Binder jetting: this method employs a powder base material and a liquid binder. In the build chamber, the powder is spread in equal layers and binder is applied through jet nozzles that "glue" the powder particles together in the shape of a programmed 3D object.

IV. Material extrusion: the most widespread and popular 3DP technology on the market. These printers are fed a thermo-plastic filament that gets pushed through a heating chamber: the fused material is moulded and then solidified through cooling, allowing the deposition of successive layers.

V. Powder bed fusion: this technology uses a high-power laser source to fuse small particles of plastic, metal, ceramic or glass powders into a mass that has the desired three-dimensional shape. The laser selectively fuses the powdered material by scanning the crosssections generated by the 3D modelling program on the surface of a powder bed.

VI. Sheet lamination: in this technique sheets of material are bound together through external force. These processes can be further categorised based on the mechanism employed to achieve bonding between layers: gluing or adhesive bonding, thermal bonding, clamping, or ultrasonic welding.

VII. Direct energy deposition: this process, mostly used in the high-tech metal industry, enables the creation of parts by melting material as it is being deposited. The 3DP is usually attached to a multi-axis robotic arm composed of a nozzle that deposits metal powder or wire on a surface and an energy source (laser, electron beam or plasma arc) that melts it, forming a solid object.

\section{Materials and methods}

All existing articles sourced through updated electronic databases (PubMed, MEDLINE, EMBASE) and published up to March 2017 were examined according to the "Preferred Reporting Items for Systematic Reviews and Meta-analyses" (PRISMA) guidelines ${ }^{11}$. The research was conducted using the following keywords: "3D printing OR three di- 

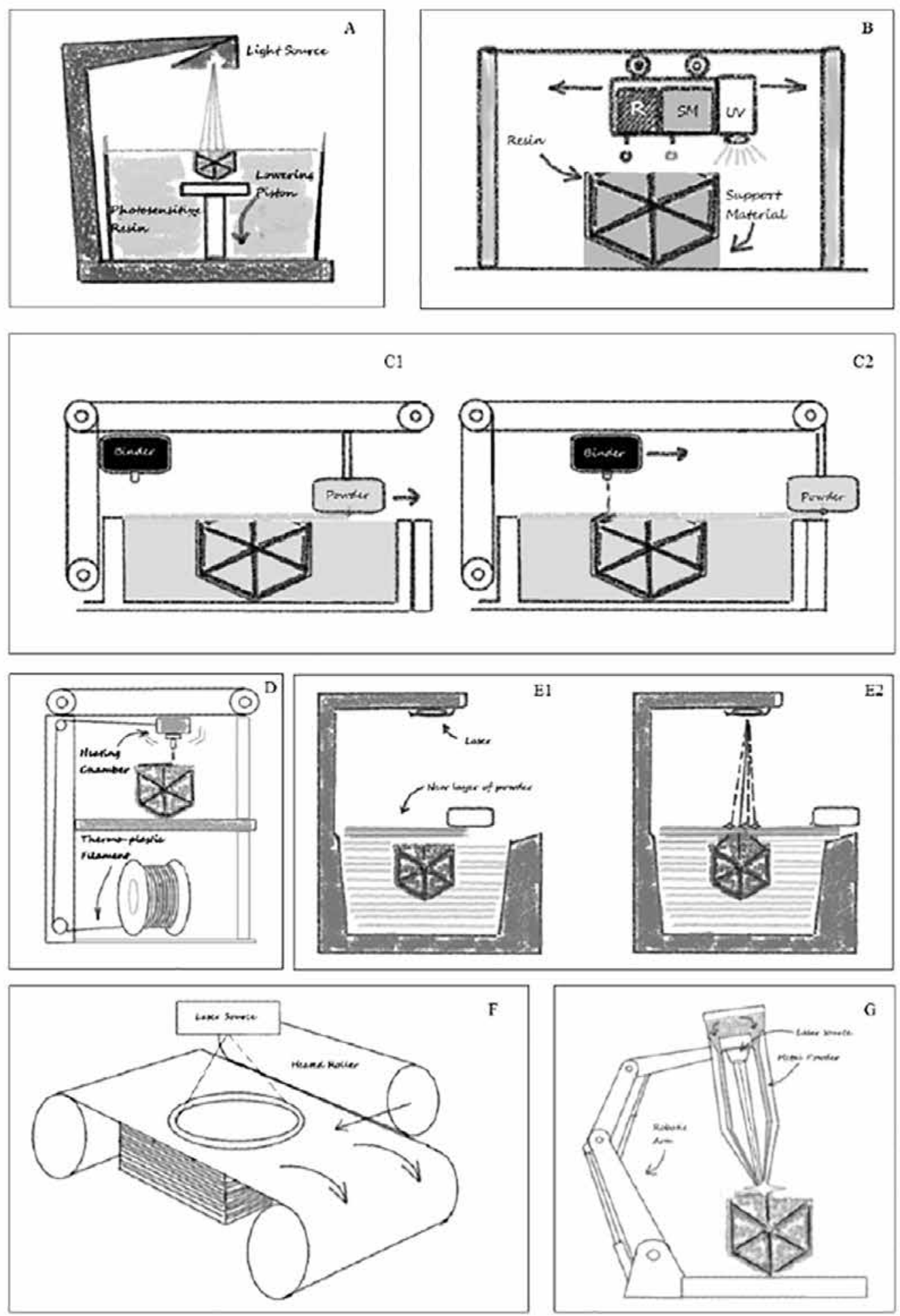

Fig. 1. Schematic representation of $A M$ technologies: $(A)$ vat photopolymerisation, (B) material jetting, $(C 1, C 2)$ binder jetting (R: resin, SM: supporting material, UV: UV lamp), (D) material extrusion, (E1, E2) powder bed fusion, (F) sheet lamination, (G) direct energy deposition. 
mensional printing AND otorhinolaryngology NOT plastic surgery", "3D printing OR three dimensional printing AND ENT NOT plastic surgery", "3D printing OR three dimensional printing AND otology NOT plastic surgery", "3D printing OR three dimensional printing AND rhinology NOT plastic surgery", "3D printing OR three dimensional printing AND head neck NOT plastic surgery", "3D Printing OR three-dimensional printing AND mandible NOT plastic surgery". Other sources analysed for additional relevant trials were reference lists of previous systematic reviews and evaluated works, journal homepages and publications citing included trials. Furthermore, experts in the field of 3D printing and engineering were contacted to ensure that all relevant studies had been included. Searches were done at all stages, from the initial drafting of the paper to submission of the revised and final version. Works lacking clinical or surgical relevance, such as engineering and bio-engineering publications and those regarding the evaluation of accuracy of the 3DP models were excluded since these are out of the expertise of ENT surgeons. Moreover, papers primarily addressing maxillofacial surgery, plastic surgery, thoracic surgery, neurosurgery and dentistry were also excluded. Exclusion criteria also applied to animal research and studies with ambiguous information regarding the modalities of production and employment of the 3DP methodology. Articles not written in English, review articles, letters, editorials and congress abstracts were omitted as well. All the considered studies were classified according to the specific field of application (otologic, rhinologic, head and neck). Each field was furthermore categorised into three distinct areas of interest: surgical and preclinical education, customised surgical planning and tissue engineering and implantable prostheses.

\section{Results}

The electronic database search yielded 258 citations and a further 123 articles were identified from additional sources, but after removing duplicates the total number of articles decreased to 278. A total of 157 records were removed as they did not fulfil inclusion criteria. Overall, 121 studies were included in the systematic review (Fig. 2). Figure 3 shows the studies according to the spe-

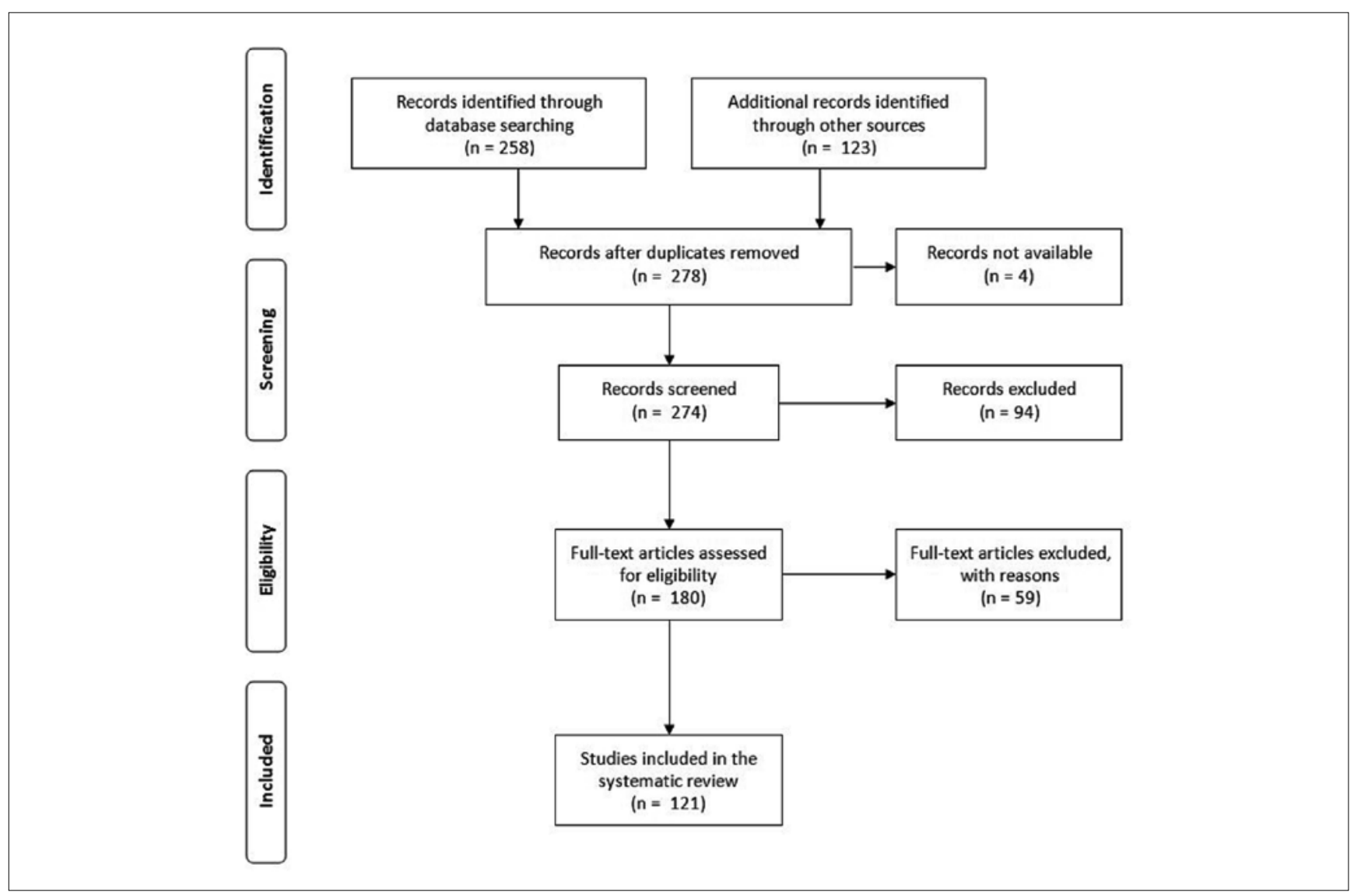

Fig. 2. PRISMA flowchart showing the study selection process. 


\section{Partition of works according to the ENT field}

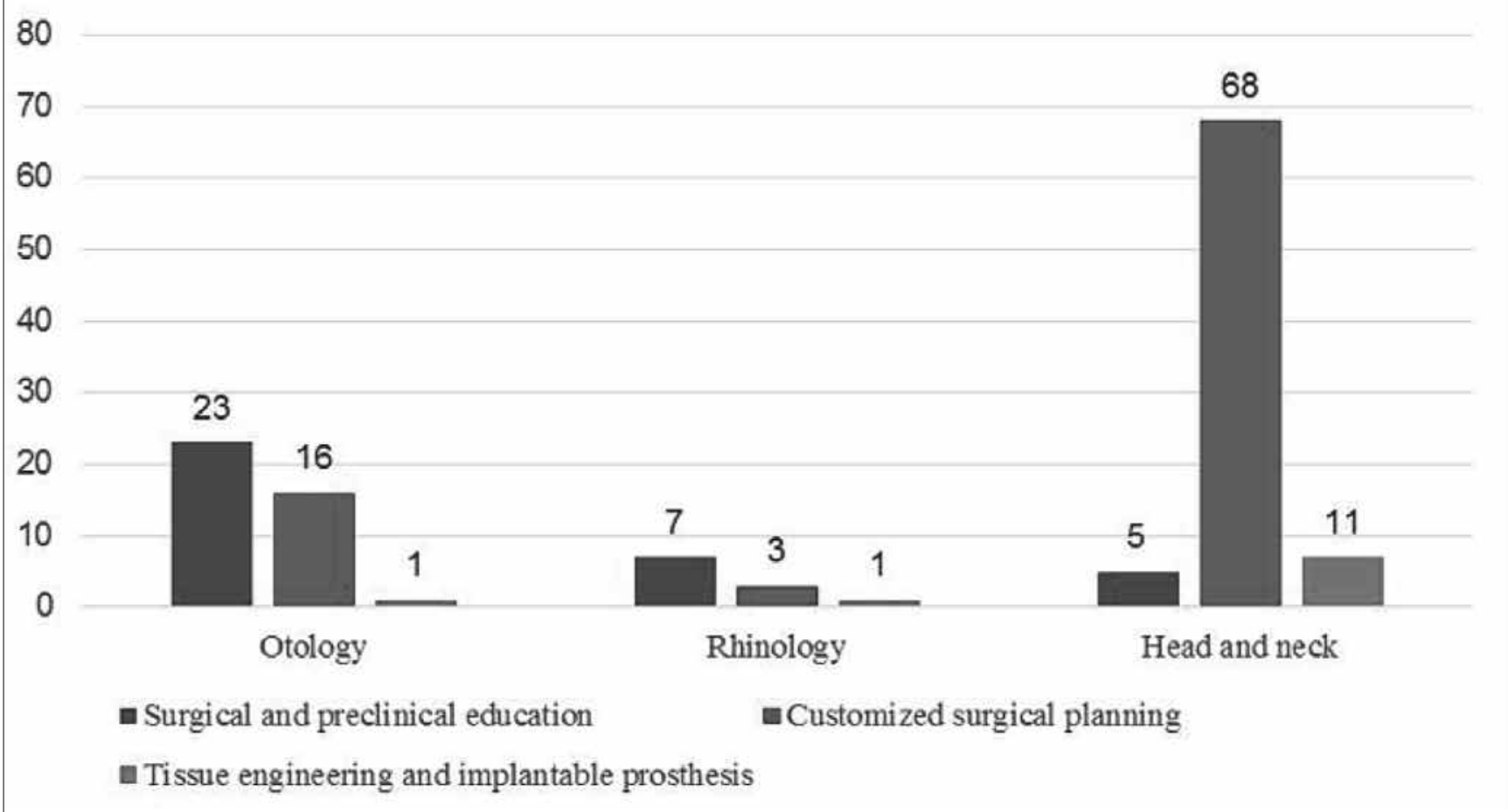

Fig. 3. Number of studies according to ENT field.

cific field of application (otologic, rhinologic, head and neck) and area of interest (surgical and preclinical education, customised surgical planning, tissue engineering and implantable prostheses). The total number of articles in Figure 3 is 135, and not 121, since 14 articles belong to more than one field of application and/or area of interest. Employed AM technology is summarised in Figure 4 considering the three areas of interest.

\section{Otologic applications (Table I)}

Surgical and preclinical education ${ }^{12-34}$

Twenty-three studies of the otologic ones $(n=39)$ involved the surgical and preclinical education area $(59.0 \%)$ and mostly concerned the field of temporal bone dissection. Since the first report in $1998^{31}$, technological efforts aimed to overcome the restrictions of the initial 3DP models. These first models, which employed a sole material and a single colour, allowed acceptable anatomical results, but limited haptic and drilling features. The evolution of 3DP systems (e.g. binder jetting) led to greater anatomical fidelity thanks to the employment of multiple colours and materials that are able to reproduce the mechanical properties of trabecular mastoid bone with realistic drilling experience. Moreover, the development of printed models coupled with electronic simulators provided a real-time alert in case of injury to vital structures during dissecting practice ${ }^{28}$.

Customised surgical planning ${ }^{2935-49}$

The production of patient-specific 3DP temporal bones based on preoperative CT was considered suitable for surgical planning and simulation in five cases of challenging anatomy (e.g. congenital aural atresia, acquired subverted anatomy) and in one case of cochlear implant surgery ${ }^{29,35-38}$. Four papers dealt with the creation of $3 \mathrm{DP}$ operative templates to assist surgical positioning of a transcutaneous bone-conduction hearing device ${ }^{39-42}$. Finally, six studies were on the combined use of surgical navigation and 3DP technology ${ }^{43-48}$. In particular, a Japanese publication described the development of a registration method based on bone-anchored fiducial markers using 3DP templates without requiring a preoperative invasive marking process or additional CT. Since its first publication, this process has been simplified and further improved.

Tissue engineering and implantable prosthetics ${ }^{50}$

Kozin et al. tested a customised 3DP prosthesis for repair of bony superior canal defects on cadaveric temporal bones, even if clinical uses were not yet reported ${ }^{50}$. 


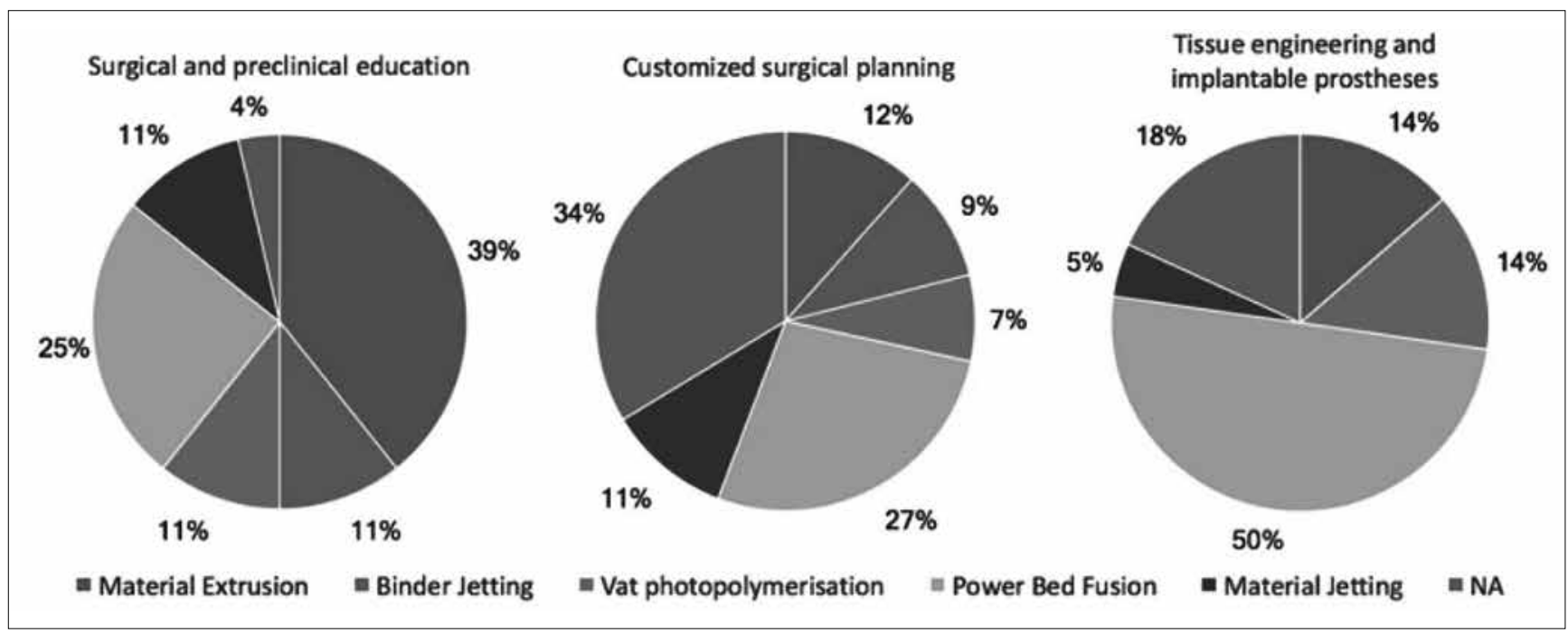

Fig. 4. Employed AM technology considering the area of interest.

Table I. Otologic studies classified according to each area of interest.

\begin{tabular}{|c|c|c|c|c|}
\hline \multicolumn{5}{|c|}{ SURGICAL AND PRECLINICAL EDUCATION } \\
\hline Field of work & Authors, year & AM category & 3D printer & 3DP material \\
\hline \multirow{20}{*}{$\begin{array}{l}\text { Temporal bone } \\
\text { dissection training } \\
\text { model }\end{array}$} & Cohen J et al., $2015^{12}$ & Material extrusion & Dimensions SST 1200es & Abs + resin (support material) \\
\hline & Da Cruz MJ et al., $2015^{13}$ & Binder jetting & Spectrum Z510 & Chalk-like powder + binder + colors \\
\hline & Hochman JB et al., 2015 (1) ${ }^{14}$ & Binder jetting & ZPrinter 650 & Chalk-like powder + binder + colors \\
\hline & Hochman JB et al., 2015 (2) ${ }^{15}$ & Binder jetting & ZPrinter 650 & Chalk-like powder + binder + colors \\
\hline & Longfield EA et al., $2015^{16}$ & Binder jetting & Spectrum Z510 & Chalk-like powder + binder + colors \\
\hline & Mowry SE et al., $2015^{17}$ & Material extrusion & MakerBot 2x & $\mathrm{ABS}+\mathrm{HIPS}$ \\
\hline & Rose AS et al., $2015^{18}$ & Vatphotopolymerisation & Objet Connex 350 & $\begin{array}{l}\text { Photo-polymer resins with different } \\
\text { mechanical properties }\end{array}$ \\
\hline & Hochman JB et al., $2014{ }^{19}$ & Binder jetting & ZPrinter 650 & Chalk-like powder + binder + colors \\
\hline & Unger BJ et al., $2014^{20}$ & Binder jetting & ZPrinter 650 & Chalk-like powder + binder + colors \\
\hline & Mick PT et al., $2013^{21}$ & Binder jetting & ZPrinter 650 & $\mathrm{Zp}^{\circledR} 131$ powder binder $\left(\mathrm{Zb}{ }^{\circledR} 7\right)+$ colors \\
\hline & Roosli C et al., 201322 & Binder jetting & Spectrum Z510 & Chalk-like powder + binder + colors \\
\hline & Bakhos D et al., $2010^{23}$ & Vat photopolymerisation & SLA ${ }^{\circledR} 5000$ & Somos $^{\circledR} 14120$ \\
\hline & Mori K, $2009^{24}$ & Powder bed fusion & $\begin{array}{l}\text { NA (commercial available } \\
\text { prototype) }\end{array}$ & Polyamide nylon and glass beads \\
\hline & Mori K et al., 200925 & Powder bed fusion & $\begin{array}{l}\text { NA (commercial available } \\
\text { prototype) }\end{array}$ & Polyamide nylon and glass beads \\
\hline & Mori K et al., $2008^{26}$ & Powder bed fusion & $\begin{array}{l}\text { NA (commercial available } \\
\text { prototype) }\end{array}$ & Polyamide nylon and glass beads \\
\hline & Suzuki M et al., $2007{ }^{27}$ & Powder bed fusion & NA & Polyamide nylon and glass beads \\
\hline & Grunert S et al., $2006^{28}$ & Binder jetting & Spectrum Z510 & $\begin{array}{l}\text { Plaster + post-processing with } \\
\text { polyurethane and acetone }\end{array}$ \\
\hline & Suzuki M et al., 2004 (1) ${ }^{29}$ & Powder bed fusion & NA & Polyamide nylon and glass beads \\
\hline & Suzuki M et al., 2004 (2) ${ }^{30}$ & Powder bed fusion & NA & Polyamide nylon and glass beads \\
\hline & Begall K et al., $1998{ }^{31}$ & Vat photopolymerisation & $\begin{array}{l}\text { Laser Model } \\
\text { stereolithographic } \\
\text { System by Fockele \& } \\
\text { Schwarze GmbH }\end{array}$ & Photosensitive; expoxy resins \\
\hline
\end{tabular}




\begin{tabular}{|c|c|c|c|c|c|}
\hline \multicolumn{6}{|c|}{ SURGICAL AND PRECLINICAL EDUCATION } \\
\hline Field of work & Authors, year & AM category & 3D printer & \multicolumn{2}{|c|}{ 3DP material } \\
\hline $\begin{array}{l}\text { Surgical middle ear } \\
\text { training model }\end{array}$ & Monfared A et al., $2012^{32}$ & Material jetting & Objet Polyjet printer & \multicolumn{2}{|c|}{ Combination of 2 photosensitive resins } \\
\hline $\begin{array}{l}\text { Endoscopic ear surgery } \\
\text { training model }\end{array}$ & Barber SR et al., $2016^{33}$ & Binder jetting & ZPrinter 650 & \multicolumn{2}{|c|}{$\begin{array}{l}\text { Zp }{ }^{\circledR} 151 \text { composite material + binder } \\
\left.\text { (ColorBond zbond }{ }^{\circledR} 90\right)+ \text { colors }\end{array}$} \\
\hline $\begin{array}{l}\text { Functioning anatomical } \\
\text { middle ear model }\end{array}$ & Kuru I et al., $2016^{34}$ & Powder bed fusion & EOS Formiga P100 & \multicolumn{2}{|c|}{ Polyamide powder PA2200 } \\
\hline \multicolumn{6}{|c|}{ CUSTOMISED SURGICAL PLANNING } \\
\hline Field of work & Authors, year & AM category & 3D printer & \multicolumn{2}{|c|}{ 3DP material } \\
\hline \multirow[t]{5}{*}{$\begin{array}{l}\text { Temporal bone surgical } \\
\text { simulation }\end{array}$} & Rose AS et al., $2015^{35}$ & Material jetting & Objet Connex 350 & \multicolumn{2}{|c|}{$\begin{array}{l}\text { Photo-polymers with different } \\
\text { mechanical properties }\end{array}$} \\
\hline & Suzuki M et al., $2005^{36}$ & Powder bed fusion & NA & \multicolumn{2}{|c|}{ Polyamide nylon and glass beads } \\
\hline & Suzuki M et al., 2004 (1) ${ }^{29}$ & Powder bed fusion & NA & \multicolumn{2}{|c|}{ Polyamide nylon and glass beads } \\
\hline & Lopponen H et al., $1997{ }^{37}$ & Vat photopolymerisation & NA & \multicolumn{2}{|c|}{ Acrylic solution } \\
\hline & Andrews JC et al., $1994^{38}$ & Vat photopolymerisation & 3D Systems SLA 250 & \multicolumn{2}{|c|}{ Liquid plastic } \\
\hline \multirow{4}{*}{$\begin{array}{l}\text { Template-guided } \\
\text { surgery }\end{array}$} & Pai I et al., $2016^{39}$ & Material jetting & Objet Eden 250 & \multicolumn{2}{|c|}{ Transparent photo-polymer } \\
\hline & Matsumoto $\mathrm{N}$ et al., $2015^{40}$ & Vat photopolymerisation & NA & \multicolumn{2}{|c|}{ Transparent photo-polymer } \\
\hline & Cho B et al., 2014"1" & Material jetting & Objet Connex 500 & \multicolumn{2}{|c|}{ Transparent photo-polymer } \\
\hline & Takumi Y et al., $2014^{42}$ & Vat photopolymerisation & NA & \multicolumn{2}{|c|}{ Transparent photo-polymer } \\
\hline \multirow[t]{7}{*}{$\begin{array}{l}\text { Navigation for } \\
\text { otoneurosurgery }\end{array}$} & Yamashita M et al., $2016^{43}$ & Material jetting & Objet Connex 500 & Phantom & $\begin{array}{l}\text { TangoPlus FLX930, } \\
\text { VeroWhitePlus } \\
\text { RGD835 }\end{array}$ \\
\hline & & & & Template & $\begin{array}{l}\text { VeroWhitePlus } \\
\text { RGD835 }\end{array}$ \\
\hline & Ritacco LE et al., $2015^{44}$ & NA & NA & \multicolumn{2}{|l|}{ NA } \\
\hline & Oka M et al., $2014{ }^{45}$ & NA & NA & \multicolumn{2}{|l|}{ NA } \\
\hline & Cho B et al., $2013^{46}$ & Powder bed fusion & NA & \multicolumn{2}{|l|}{ NA } \\
\hline & Matsumoto N et al., $2012^{47}$ & Powder bed fusion & NA & \multicolumn{2}{|l|}{ NA } \\
\hline & Matsumoto N et al., 200948 & Powder bed fusion & NA & \multicolumn{2}{|l|}{ NA } \\
\hline $\begin{array}{l}\text { Lateral } \\
\text { skull base approaches }\end{array}$ & Muelleman TJ et al., $2016^{49}$ & Material extrusion & uPrint SE Plus & \multicolumn{2}{|c|}{ Thermo-plastic material } \\
\hline \multicolumn{6}{|c|}{ TISSUE ENGINEERING AND IMPLANTABLE PROSTHESIS } \\
\hline Field of work & Authors, year & AM category & 3D printer & \multicolumn{2}{|c|}{ 3DP material } \\
\hline $\begin{array}{l}\text { Prosthesis for superior } \\
\text { canal dehiscence }\end{array}$ & Kozin ED et al., $2015^{50}$ & $\begin{array}{l}\text { Vat photopolymerisation } \\
\text { Powder bed fusion }\end{array}$ & $\begin{array}{l}\text { FormLabs Form 1+ } \\
\text { EOS Formiga }\end{array}$ & \multicolumn{2}{|c|}{$\begin{array}{l}\text { Plastic-based material; Aluminium- } \\
\text { based material }\end{array}$} \\
\hline
\end{tabular}

ABS: Acrylonitrile Butadiene Styrene; PLA: PolyLactic Acid; HIPS: High Impact PolyStyrene; NA: not available.

\section{Rhinologic applications (Table II)}

Surgical and preclinical education ${ }^{51-57}$

Four studies focused on the development of 3DP training models for endoscopic sinonasal and skull base surgery ${ }^{51-54}$. Medium-high fidelity simulators allowed developing surgical skills in the main endoscopic procedures, including drilling techniques and skull base exposure. Low-cost models were primary limited by the materials employed to mimic human bone as much as possible.
Customised surgical planning ${ }^{58-60}$

Two studies took advantage of the versatility of 3DP systems to fabricate operative templates tailored on the patient's anatomy. Daniel et al. produced 3DP cutting guides to design an osteoplastic flap during frontal surgery ${ }^{59}$; Onerci Altunay et al. used 3DP templates to fashion septal prosthesis for large irregular septal perforations ${ }^{58}$. 3DP endoscopic sinus surgery simulation was carried out in two patients with chronic rhinosinusitis to obtain safer and faster procedures ${ }^{60}$. 
Table II. Rhinologic studies classified according to each area of interest.

\begin{tabular}{|c|c|c|c|c|c|}
\hline \multicolumn{6}{|c|}{ SURGICAL AND PRECLINICAL EDUCATION } \\
\hline Field of work & \multicolumn{2}{|l|}{ Authors, year } & AM category & 3D printer & 3DP material \\
\hline \multirow{6}{*}{$\begin{array}{l}\text { Endoscopic sinonasal } \\
\text { and skull base training } \\
\text { models }\end{array}$} & \multicolumn{2}{|c|}{ Chang DR et al., $2017^{51}$} & Material extrusion & Airwolf 3D HD2X & $\begin{array}{l}\text { ABS + molding with Aquasil Ultra XLV } \\
\text { silicone }\end{array}$ \\
\hline & \multicolumn{2}{|c|}{ Tai BL et al., $2016{ }^{52}$} & Material extrusion & NA & Thermo-plastic material \\
\hline & \multicolumn{2}{|c|}{ Narayanan V et al., $2015^{53}$} & Material jetting & Objet Connex 500 & $\begin{array}{l}\text { Photo-polymers with different } \\
\text { mechanical properties }\end{array}$ \\
\hline & \multirow[t]{3}{*}{$\begin{array}{l}\text { Chan HHL et al., } \\
2015^{54}\end{array}$} & $\begin{array}{l}\text { Paranasal } \\
\text { sinus } \\
\text { phantom }\end{array}$ & Material extrusion & Vantage - Stratasys & ABS \\
\hline & & $\begin{array}{l}\text { Skull base } \\
\text { phantom }\end{array}$ & Binder jetting & ZPrinter 310 - ZCorp & $\begin{array}{l}\text { ZP-130 plaster powder + CA101 } \\
\text { cyanoacrylate; ZP-15 plaster powder + } \\
\text { infiltrant elastomeric }\end{array}$ \\
\hline & & $\begin{array}{l}\text { Mandible } \\
\text { templates }\end{array}$ & Material extrusion & Vantage - Stratasys & Polycarbonate \\
\hline $\begin{array}{l}\text { Septoplasty training } \\
\text { model }\end{array}$ & \multicolumn{2}{|c|}{ AlReefi MA et al., $2017^{55}$} & Material jetting & Objet Connex 500 & $\begin{array}{l}\text { VeroWhitePlus, Tango- } \\
\text { Plus and their combination to simulate } \\
\text { different mechanical properties }\end{array}$ \\
\hline $\begin{array}{l}\text { Nosebleed training } \\
\text { model }\end{array}$ & \multicolumn{2}{|c|}{ Estomba C et al., $2016^{56}$} & NA & NA & PLA + Polyurethane \\
\hline Anatomical models & \multicolumn{2}{|c|}{ Sander IM et al., $2017^{57}$} & Material extrusion & LulzBot TAZ 5 & PLA \\
\hline \multicolumn{6}{|c|}{ CUSTOMISED SURGICAL PLANNING } \\
\hline Field of work & \multicolumn{2}{|l|}{ Authors, year } & AM category & 3D printer & 3DP material \\
\hline \multirow{2}{*}{$\begin{array}{l}\text { Template-guided } \\
\text { surgery }\end{array}$} & \multicolumn{2}{|c|}{ Onerci Altunay Z et al., $2016{ }^{58}$} & Binder jetting & Spectrum Z510 & Z131 powder \\
\hline & \multicolumn{2}{|c|}{ Daniel M et al., $2011^{59}$} & Binder jetting & ZPrinter 310 plus & NA \\
\hline $\begin{array}{l}\text { Endoscopic sinus } \\
\text { surgery simulation }\end{array}$ & \multicolumn{2}{|c|}{ Raos P et al., $2015^{60}$} & Binder jetting & ZPrint 310 & NA \\
\hline \multicolumn{6}{|c|}{ TISSUE ENGINEERING AND IMPLANTABLE PROSTHESIS } \\
\hline Field of work & \multicolumn{2}{|l|}{ Authors, year } & AM category & 3D printer & 3DP material \\
\hline Customised prosthesis & \multicolumn{2}{|c|}{ Nahumi N et al., $2015^{61}$} & NA & NA & PolyEtherEtherKetone \\
\hline
\end{tabular}

ABS: Acrylonitrile Butadiene Styrene; PLA: PolyLactic Acid; HIPS: High Impact PolyStyrene; NA: not available.

Tissue engineering and implantable prosthetics ${ }^{61}$

One child with a craniofacial fibrous dysplasia was submitted to resection and reconstruction of the fronto-orbital region by means of a custom 3DP polyetheretherketone implant resulting in good aesthetical and safe outcomes.

\section{Head and neck applications (Table III)}

Surgical and preclinical education ${ }^{62-66}$ :

Two studies focused on resident training for laryngeal surgical procedures. In 2014, Ainsworth et al. created a laryngeal model, including the extra-laryngeal soft tissues, to simulate trans-cervical injection of vocal folds ${ }^{64}$. More recently, Kavanagh et al. developed a 3DP paediatric laryngeal model reproducing several challenging surgical conditions (e.g. subglottic cysts, laryngomalacia, subglottic stenosis and laryngeal clefts) ${ }^{62}$.
Customised surgical planning 5465 67-132

This was the most frequent ENT application of 3DP technology and mentioned in 68 of the 121 papers $(56.2 \%)$. Among these, $95.6 \%$ of studies (65 out of 68$)^{5467-130}$ concerned surgical management of head and neck tumours requiring mandibular resection and/or reconstruction. The first date to the '90s and dealt with creation of 3DP mandibles to allow a direct handling of the neoplastic lesion, leading to the early surgical resection simulators. However, the most relevant contribution concerned the reconstructive aspects of oncologic surgery, guiding the employment of plates or autografts. Patient-specific 3DP mandibles were developed to "pre-bent" plates preoperatively. More recently, the introduction of image-guide systems used to plan the harvest and positioning of autografts (e.g. fibula flap, iliac crest bone flap) has led to the production of self-fabricated 
Table III. Head and neck studies classified according to each area of interest.

\begin{tabular}{|c|c|c|c|c|c|}
\hline \multicolumn{6}{|c|}{ SURGICAL AND PRECLINICAL EDUCATION } \\
\hline Field of work & \multicolumn{2}{|c|}{ Authors, year } & AM category & 3D printer & 3DP material \\
\hline \multirow[t]{3}{*}{ Laryngeal model } & \multicolumn{2}{|c|}{ Kavanagh KR et al., $2017^{62}$} & Material extrusion & MakerBot & ABS, PLA, HIPS \\
\hline & \multicolumn{2}{|c|}{ Johnson CM et al., $2016{ }^{63}$} & Material extrusion & MakerBot 2XL & $\begin{array}{l}\text { ABS (best performance), HIPS, PLA; } \\
\text { Dragon Skin Fast silicon casting in a } \\
\text { 3D printed mold }\end{array}$ \\
\hline & \multicolumn{2}{|c|}{ Ainsworth TA et al., $2014^{64}$} & Material extrusion & Dimension Elite - Stratasys & ABSplus + silicone casting \\
\hline Carotid artery model & \multicolumn{2}{|c|}{ Govsa F et al., $2017{ }^{65}$} & Material extrusion & MakerBot & PLA \\
\hline Tracheostoma model & \multicolumn{2}{|c|}{ Grolman W et al., $1995^{66}$} & Vat photopolimerisation & NA & Synthetic liquid resin \\
\hline \multicolumn{6}{|c|}{ CUSTOMISED SURGICAL PLANNING } \\
\hline Field of work & \multicolumn{2}{|l|}{ Authors, year } & AM category & 3D printer & 3DP material \\
\hline \multirow[t]{23}{*}{$\begin{array}{l}\text { Guided surgery for oro- } \\
\text { mandibular resection } \\
\text { and reconstruction }\end{array}$} & \multicolumn{2}{|c|}{ Bosc $R$ et al., $2017^{67}$} & $\begin{array}{l}\text { Material jetting } \\
\text { Material extrusion }\end{array}$ & $\begin{array}{l}\text { Objet 30Pro - Stratasys } \\
\text { Zortrax M200 - Zortrax } \\
\text { SARL }\end{array}$ & $\begin{array}{l}\text { Biocompatible photopolymer } \\
\text { ABS }\end{array}$ \\
\hline & $\begin{array}{l}\text { Rachmiel A et al., } \\
2017^{68}\end{array}$ & Skull & Material jetting & Objet260 Dental - Stratasys & Photopolimer resin \\
\hline & & Template & Powder bed fusion & EOS & Titanium \\
\hline & \multicolumn{2}{|c|}{ Shah S et al., $2017^{69}$} & Binder jetting & ZPrinter 310 plus & Gypsum-based material \\
\hline & \multicolumn{2}{|c|}{ Lee UL et al., $2016{ }^{70}$} & Powder bed fusion & $\begin{array}{l}\text { Arcam A1 (Electron Beam } \\
\text { Melting) }\end{array}$ & Ti-6Al-4 V-ELI medical grade powder \\
\hline & \multirow[t]{2}{*}{$\begin{array}{l}\text { Lim SH et al., } \\
2016^{71}\end{array}$} & Mandible & Binder jetting & ProJet 360 - 3D Systems & NA \\
\hline & & $\begin{array}{l}\text { Cutting/ } \\
\text { position- } \\
\text { ing } \\
\text { guides }\end{array}$ & Material jetting & $\begin{array}{l}\text { ProJet } 3500 \\
\text { HDMax - 3D Systems }\end{array}$ & Biocompatible materials \\
\hline & \multicolumn{2}{|c|}{ Numajiri T et al., $2016^{72}$} & Material extrusion & MakerBot & PLA \\
\hline & \multicolumn{2}{|c|}{ Yamada H et al., $2016^{73}$} & NA & NA & NA \\
\hline & \multirow[t]{3}{*}{$\begin{array}{l}\text { Chan HHL et al., } \\
2015^{54}\end{array}$} & $\begin{array}{l}\text { Paranasal } \\
\text { sinus } \\
\text { phantom }\end{array}$ & Material extrusion & Vantage - Stratasys & ABS \\
\hline & & $\begin{array}{l}\text { Skull base } \\
\text { phantom }\end{array}$ & Binder jetting & ZPrinter 310 - ZCorp & $\begin{array}{l}\text { ZP-130 plaster powder + CA101 } \\
\text { cyanoacrylate; ZP-15 plaster powder + } \\
\text { infiltrant elastomeric }\end{array}$ \\
\hline & & $\begin{array}{l}\text { Mandible } \\
\text { templates }\end{array}$ & Material extrusion & Vantage - Stratasys & Polycarbonate \\
\hline & \multicolumn{2}{|c|}{ Man QW et al., $2015^{74}$} & NA & NA & NA \\
\hline & \multicolumn{2}{|c|}{ Modabber A et al., $2015^{75}$} & Powder bed fusion & NA & Polyamide Powder \\
\hline & \multicolumn{2}{|c|}{ Reiser V et al., $2015^{76}$} & Material jetting & $\begin{array}{l}\text { A Objet - Stratasys } \\
\text { machine (Model NA) }\end{array}$ & $\begin{array}{l}\text { Biocompatible plastic } \\
\text { polymers }\end{array}$ \\
\hline & \multicolumn{2}{|c|}{ Schepers RH et al., $2015^{77}$} & NA & NA & Polyamide (for the cutting guides) \\
\hline & \multirow[t]{2}{*}{$\begin{array}{l}\text { Shan XF et al., } \\
2015^{78}\end{array}$} & $\begin{array}{l}\text { Residual } \\
\text { skull }\end{array}$ & Material extrusion & Stratasys FDM 400-mc & NA \\
\hline & & Mesh & NA & NA & Titanium \\
\hline & \multicolumn{2}{|c|}{ Steinbacher DM et al., $2015^{79}$} & NA & & \\
\hline & \multicolumn{2}{|c|}{ Succo G et al., $2015^{80}$} & NA & NA & NA \\
\hline & \multicolumn{2}{|c|}{ Wilde F et al., $2015^{81}$} & Powder bed fusion & NA & Polyamide \\
\hline & \multicolumn{2}{|c|}{ Ayoub N et al., $2014^{82}$} & Powder bed fusion & NA & NA \\
\hline & \multicolumn{2}{|c|}{ Azuma M et al., $2014^{83}$} & Binder jetting & ZPrinter 310 plus & NA \\
\hline
\end{tabular}


Table III. follows

\begin{tabular}{|c|c|c|c|c|c|}
\hline \multicolumn{6}{|c|}{ CUSTOMISED SURGICAL PLANNING } \\
\hline Field of work & \multicolumn{2}{|l|}{ Authors, year } & AM category & 3D printer & 3DP material \\
\hline & \multicolumn{2}{|c|}{ de Farias TP et al., $2014{ }^{84}$} & Binder jetting & Z-Corp Spectrum Z510 & $\begin{array}{l}\text { Gypsum, cyanoacrylate, } \\
\text { and ZP150 }\end{array}$ \\
\hline & \multicolumn{2}{|c|}{ Liu YF et al., $2014{ }^{85}$} & Powder bed fusion & $\begin{array}{l}\text { Sinterstation HiQ +HiSTM - } \\
\text { 3D Systems }\end{array}$ & DuraForm - biocompatible nylon \\
\hline & \multicolumn{2}{|c|}{ Modabber A et al., $2014{ }^{86}$} & Powder bed fusion & NA & Polyamide \\
\hline & \multicolumn{2}{|c|}{ Tsai MJ et al., $2014^{87}$} & NA & NA & NA \\
\hline & \multicolumn{2}{|c|}{ Watson J et al., $2014{ }^{88}$} & Powder bed fusion & $\begin{array}{l}\text { Direct metal Powder bed } \\
\text { fusion (Model NA) }\end{array}$ & $\begin{array}{l}\text { Medical-grade titanium alloy Ti6AL4V } \\
\text { - 3TRPD }\end{array}$ \\
\hline & \multicolumn{2}{|c|}{ Wilde F et al., $2014{ }^{89}$} & Powder bed fusion & NA & Biocompatible Polyamide \\
\hline & \multicolumn{2}{|c|}{ Yamada H et al., $2014{ }^{90}$} & NA & NA & NA \\
\hline & \multicolumn{2}{|c|}{ Coppen C et al., $2013^{91}$} & Powder bed fusion & NA & DuraForm PA - 3DWorknet \\
\hline & \multicolumn{2}{|c|}{ Foley BD et al., $2013^{92}$} & NA & NA & NA \\
\hline & \multicolumn{2}{|c|}{ Hanasono MM et al., $2013^{93}$} & NA & NA & NA \\
\hline & \multirow[t]{3}{*}{$\begin{array}{l}\text { Mazzoni S et al., } \\
2013^{94}\end{array}$} & Plate & Powder bed fusion & $\begin{array}{l}\text { EOSINT M270 - Electro- } \\
\text { Optical Systems }\end{array}$ & EOS Titanium Ti64 \\
\hline & & Guide & Powder bed fusion & $\begin{array}{l}\text { EOSINT M270 - Electro- } \\
\text { Optical Systems }\end{array}$ & EOS Cobalt-Chrome MP1 \\
\hline & & Mandible & NA & Stratasys machine & Resin \\
\hline & \multicolumn{2}{|c|}{ Zheng GS et al., $2013^{95}$} & Vat photopolymerisation & SLA-3500 3D Systems & NA \\
\hline & \multirow[t]{3}{*}{$\begin{array}{l}\text { Ciocca L et al., } \\
2012(1)^{96}\end{array}$} & Plate & Powder bed fusion & $\begin{array}{l}\text { EOSINT M270 - Electro- } \\
\text { Optical Systems }\end{array}$ & EOS Titanium Ti64 \\
\hline & & Guide & Powder bed fusion & $\begin{array}{l}\text { EOSINT M270 - Electro- } \\
\text { Optical Systems }\end{array}$ & EOS Cobalt-Chrome MP1 \\
\hline & & Mandible & Material extrusion & Stratasys machine & ABS \\
\hline & \multirow[t]{3}{*}{$\begin{array}{l}\text { Ciocca L et al., } \\
2012(2)^{97}\end{array}$} & Plate & Powder bed fusion & $\begin{array}{l}\text { EOSINT M270 - Electro- } \\
\text { Optical Systems }\end{array}$ & EOS Titanium Ti64 \\
\hline & & Guide & Powder bed fusion & $\begin{array}{l}\text { EOSINT M270 - Electro- } \\
\text { Optical Systems }\end{array}$ & EOS Cobalt-Chrome MP1 \\
\hline & & Mandible & Material extrusion & Stratasys machine & ABS \\
\hline & \multicolumn{2}{|c|}{ Dérand P et al., $2012^{98}$} & Powder bed fusion & ARCAM EBM A2 & Ti6Al64V ELI powder \\
\hline & \multicolumn{2}{|c|}{ Hou JS et al., $2012^{99}$} & NA & NA & Photopolymer \\
\hline & \multicolumn{2}{|c|}{ Lethaus B et al., $2012{ }^{100}$} & Material extrusion & Maastricht Instruments & NA \\
\hline & \multirow[t]{2}{*}{$\begin{array}{l}\text { Modabber A et } \\
\text { al., } 2012 \text { (1) }{ }^{101}\end{array}$} & Guide & Powder bed fusion & NA & Polyamide \\
\hline & & Skull & NA & NA & Acrylic Resin \\
\hline & \multirow[t]{2}{*}{$\begin{array}{l}\text { Modabber A et } \\
\text { al., } 2012(2){ }^{102}\end{array}$} & Guide & Powder bed fusion & NA & Polyamide \\
\hline & & Skull & NA & NA & NA \\
\hline & \multicolumn{2}{|c|}{ Patel A et al., $2012^{103}$} & NA & NA & NA \\
\hline & \multicolumn{2}{|c|}{ Sink J et al., $2012^{104}$} & NA & NA & NA \\
\hline & \multicolumn{2}{|c|}{ Wilde F et al., $2012^{105}$} & Binder jetting & ZTM 510 - 4D Concepts & NA \\
\hline & \multicolumn{2}{|c|}{ Zheng GS et al., $2012{ }^{106}$} & Vat photopolymerisation & SLA-3500 3D Systems & NA \\
\hline & \multicolumn{2}{|c|}{ Abou-ElFetouh A et al., $20111^{107}$} & $\begin{array}{l}\text { Vat photopolymerisation } \\
\text { Binder jetting }\end{array}$ & $\begin{array}{l}\text { 3D Systems InVision Si2 } \\
\text { 3D Systems VisiJet SR } 200\end{array}$ & $\begin{array}{l}\text { NA } \\
\text { NA }\end{array}$ \\
\hline & \multicolumn{2}{|c|}{ Antony AK et al., $2011{ }^{108}$} & NA & NA & NA \\
\hline & Bell RB et al., 201 & 1109 & NA & NA & Acrylic resin \\
\hline & Hou JS et al., 201 & 1110 & NA & NA & Polybutadiene-styrene resin \\
\hline & Mehra Pet al., 201 & $1^{111}$ & $\begin{array}{l}\text { Vat photopolymerisation } \\
\text { Material extrusion }\end{array}$ & NA & $\begin{array}{l}\text { Acrylic, Epoxy } \\
\text { Starch }\end{array}$ \\
\hline
\end{tabular}




\begin{tabular}{|c|c|c|c|c|c|}
\hline \multicolumn{6}{|c|}{ CUSTOMISED SURGICAL PLANNING } \\
\hline Field of work & \multicolumn{2}{|l|}{ Authors, year } & AM category & 3D printer & 3DP material \\
\hline & \multicolumn{2}{|c|}{ Yamanaka Y et al., $2010^{112}$} & NA & NA & Acrylic plastic \\
\hline & \multicolumn{2}{|c|}{ Zhou LB et al., $2010^{113}$} & Vat photopolymerisation & LPS 600 laser prototyping & Resin \\
\hline & \multicolumn{2}{|c|}{ Cohen A et al., $2009{ }^{114}$} & Material jetting & Eden $500 \mathrm{~V}$ & Photo-polymer \\
\hline & \multicolumn{2}{|c|}{ Farina R et al., $2009{ }^{115}$} & $\begin{array}{l}\text { Vat photopolymerisation } \\
\text { Binder jetting }\end{array}$ & $\begin{array}{l}\text { 3D Systems SLA-250/30 } \\
\text { Z-Corporation Z406 }\end{array}$ & $\begin{array}{l}8110 \text { resin (DSM Somos) } \\
\text { Starch-cellulose material }\end{array}$ \\
\hline & \multicolumn{2}{|c|}{ Juergens P et al., 2009116} & NA & NA & NA \\
\hline & \multicolumn{2}{|c|}{ Leiggener C et al., 2009117} & Powder bed fusion & NA & Medical grade polyamide \\
\hline & \multicolumn{2}{|c|}{ Liu XJ et al., 2009118} & NA & NA & Resin \\
\hline & \multicolumn{2}{|c|}{ Chow LK et al., $2007^{119}$} & NA & NA & Starch, epoxy resin, acrylic \\
\hline & \multicolumn{2}{|c|}{ Lee JW et al., $2007^{120}$} & NA & NA & NA \\
\hline & \multicolumn{2}{|c|}{ Ro EY et al., $2007^{121}$} & NA & NA & Epoxy \\
\hline & \multicolumn{2}{|c|}{ Toro C et al., $2007^{122}$} & Vat photopolymerisation & SLA 3500 - 3D Systems & Epoxy resin Watershed 11120 \\
\hline & \multicolumn{2}{|c|}{ Yeung RWK et al,. $2007^{123}$} & NA & NA & NA \\
\hline & \multicolumn{2}{|c|}{ Hallermann W et al., $2006^{124}$} & Powder bed fusion & NA & $\begin{array}{l}\text { Duraform } \\
\text { PA12 - 3D Systems }\end{array}$ \\
\hline & \multicolumn{2}{|c|}{ Hannen EJM et al., $2006{ }^{125}$} & NA & NA & Resin \\
\hline & \multicolumn{2}{|c|}{$\begin{array}{l}\text { Cunningham LL et al., } \\
2005^{126}\end{array}$} & $\begin{array}{l}\text { Vat photopolymerisation } \\
\text { Binder jetting }\end{array}$ & $\begin{array}{l}\text { 3D Systems SLA-250/30 } \\
\text { Z-Corporation Z406 }\end{array}$ & $\begin{array}{l}8110 \text { resin (DSM Somos) } \\
\text { Starch-cellulose material }\end{array}$ \\
\hline & \multicolumn{2}{|c|}{ Wong TY et al., $2005^{127}$} & NA & NA & NA \\
\hline & \multicolumn{2}{|c|}{ Singare S et al., $2004{ }^{128}$} & Vat photopolymerisation & LPS 600 & Photo-polymer \\
\hline & \multicolumn{2}{|c|}{ Kernan BT et al., $2000{ }^{129}$} & NA & NA & NA \\
\hline & \multicolumn{2}{|c|}{ Komori T et al., $1994{ }^{130}$} & Vat photopolymerisation & $\begin{array}{l}\text { Solid Creation System } \\
\text { (D-MEC Ltd, Tokyo, Japan), }\end{array}$ & Desolight SCR- 100, D-MEC Ltd) \\
\hline $\begin{array}{l}\text { Guided surgery for } \\
\text { cranio-cervicofacial } \\
\text { teratoma }\end{array}$ & \multicolumn{2}{|c|}{$\begin{array}{l}\text { Wiedermann JP et al., } \\
2017^{131}\end{array}$} & NA & NA & NA \\
\hline Carotid artery model & \multicolumn{2}{|c|}{ Govsa F et al., 201765} & Material extrusion & MakerBot & PLA \\
\hline $\begin{array}{l}\text { MRI compatible } \\
\text { laryngoscope }\end{array}$ & \multicolumn{2}{|c|}{ Paydarfar JA et al., $2016^{132}$} & Material jetting & Objet Eden250 - Stratasys & $\begin{array}{l}\text { MED610 (Stratasys) biocompatible } \\
\text { photopolymer }\end{array}$ \\
\hline \multicolumn{6}{|c|}{ TISSUE ENGINEERING AND IMPLANTABLE PROSTHESIS } \\
\hline Field of work & \multicolumn{2}{|c|}{ Authors, year } & AM category & 3D printer & 3DP material \\
\hline \multirow{10}{*}{$\begin{array}{l}\text { Customised prosthesis } \\
\text { for mandibular } \\
\text { reconstruction }\end{array}$} & $\begin{array}{l}\text { Rachmiel A et al., } \\
201768\end{array}$ & Skull & Material jetting & Objet260 Dental - Stratasys & Photopolymer resin \\
\hline & & Template & Powder bed fusion & EOS & Titanium \\
\hline & Lee UL et al., 2016 & & Powder bed fusion & $\begin{array}{l}\text { Arcam A1 (Electron Beam } \\
\text { Melting) }\end{array}$ & Ti-6Al-4 V-ELI medical grade powder \\
\hline & Schepers RH et al. & $2015^{77}$ & NA & NA & Polyamide (for the cutting guides) \\
\hline & $\begin{array}{l}\text { Shan XF et al., } \\
2015^{78}\end{array}$ & $\begin{array}{l}\text { Residual } \\
\text { Skull }\end{array}$ & Material extrusion & Stratasys FDM 400-mc & NA \\
\hline & & Mesh & NA & NA & Titanium \\
\hline & $\begin{array}{l}\text { Watson J et al., } \\
2014{ }^{88}\end{array}$ & & Powder bed fusion & $\begin{array}{l}\text { Direct metal Powder bed } \\
\text { fusion (Model NA) }\end{array}$ & $\begin{array}{l}\text { Medical-grade titanium alloy Ti6AL4V } \\
\text { - 3TRPD }\end{array}$ \\
\hline & $\begin{array}{l}\text { Mazzoni S et al., } \\
2013^{94}\end{array}$ & Plate & Powder bed fusion & $\begin{array}{l}\text { EOSINT M270 - Electro- } \\
\text { Optical Systems }\end{array}$ & EOS Titanium Ti64 \\
\hline & & Guide & Powder bed fusion & $\begin{array}{l}\text { EOSINT M270 - Electro- } \\
\text { Optical Systems }\end{array}$ & EOS Cobalt-Chrome MP1 \\
\hline & & Mandible & NA & Stratasys machine & Resin \\
\hline
\end{tabular}




\begin{tabular}{|c|c|c|c|c|c|}
\hline \multicolumn{6}{|c|}{ TISSUE ENGINEERING AND IMPLANTABLE PROSTHESIS } \\
\hline \multirow{10}{*}{ Field of work } & \multicolumn{2}{|l|}{ Authors, year } & \multirow{2}{*}{$\begin{array}{l}\text { AM category } \\
\text { Powder bed fusion }\end{array}$} & \multirow{2}{*}{$\begin{array}{l}\text { 3D printer } \\
\text { EOSINT M270 - Electro- } \\
\text { Optical Systems }\end{array}$} & \multirow{2}{*}{$\begin{array}{l}\text { 3DP material } \\
\text { EOS Titanium Ti64 }\end{array}$} \\
\hline & $\begin{array}{l}\text { Ciocca L et al., } \\
2012 \text { (1) }{ }^{96}\end{array}$ & Plate & & & \\
\hline & & Guide & Powder bed fusion & $\begin{array}{l}\text { EOSINT M270 - Electro- } \\
\text { Optical Systems }\end{array}$ & EOS Cobalt-Chrome MP1 \\
\hline & & Mandible & Material Extrusion & Stratasys machine & ABS \\
\hline & $\begin{array}{l}\text { Ciocca L et al., } \\
2012(2)^{97}\end{array}$ & Plate & Powder bed fusion & $\begin{array}{l}\text { EOSINT M270 - Electro- } \\
\text { Optical Systems }\end{array}$ & E0S Titanium Ti64 \\
\hline & & Guide & Powder bed fusion & $\begin{array}{l}\text { EOSINT M270 - Electro- } \\
\text { Optical Systems }\end{array}$ & EOS Cobalt-Chrome MP1 \\
\hline & & Mandible & Material extrusion & Stratasys machine & ABS \\
\hline & Dérand P et al., & $12^{98}$ & Powder bed fusion & ARCAM EBM A2 & Ti6Al64V ELI powder \\
\hline & Zhou LB et al., 2 & 0113 & Vat photopolymerisation & LPS 600 laser prototyping & Resin \\
\hline & Singare $S$ et al., & $04^{128}$ & Vat photopolymerisation & LPS 600 & Photopolymer \\
\hline
\end{tabular}

ABS: Acrylonitrile Butadiene Styrene; PLA: PolyLactic Acid; HIPS: High Impact PolyStyrene; NA: not available.

customised 3DP cutting guides. Many authors experienced a decrease in surgical time and the risk of undesirable events during reconstructive approaches, which resulted in a proper mandibular function. Concerning AM technology, in $38.2 \%$ of the studies ( 26 of 68 ) the AM category was not specified, mainly due to the outsourcing of all 3D printing operations to external services, which are becoming more common in recent years.

\section{Tissue engineering and implantable}

prosthetics $68707778889496-98113128$

This area included $9.1 \%$ of all studies (11 of 121). All these investigations dealt with mandibular reconstruction following tumour resection in a total of 33 patients. The authors employed 3DP technology to develop patient-specific reconstruction plates, trays, meshes and mandibular implants. Titanium alloys (e.g. Ti6Al4V) were used in all cases due to their suitable physical and mechanical properties: low specific weight, corrosion resistance and good biocompatibility ${ }^{96}$. 3DP reconstruction plates, tray and meshes were associated with a bone autograft in 9 studies: $66.6 \%$ opted for a fibula free flap $77789496-98$ and $33.3 \%$ for an iliac crest free flap ${ }^{68} 113128$. Differently, Lee et al. made use of a mandibular implant without the support of a bone autograft, proving an acceptable alternative in cases of unsuitable free flap surgery ${ }^{70}$. A total of 27 patients $(81.9 \%)$ showed good aesthetical and occlusion outcomes and thus correct oral rehabilitation 6870777888949697113128 . Complications were observed in 2 subjects $(6 \%)$ : one patient experienced bone resorption and infection, while the other had flap necrosis ${ }^{77113}$. The authors reported a reduction of the operating time between $30{ }^{98}$ and 120 minutes ${ }^{94}$, enabling economic benefits at the expense of the additional cost of the 3DP prosthesis.

\section{Discussion}

Personalised medicine, minimally-invasive surgery, tissue engineering and regenerative medicine are the watchwords of third millennium healthcare. The arising popularity around the world of 3DP systems may be explained through the opportunities offered by this new technology to support new trends in modern medicine. Since its first applications in the early 1990s, researchers have explored the advantages of $3 \mathrm{D}$ printers, publishing 121 studies in otorhinolaryngology (Fig. 2). Customised surgical planning was evaluated in $71.9 \%$ of studies, proving to be the main direction of investigation (Fig. 3). The manufacture of anatomical models before surgery allowed both the understanding of specific anomalies and guidance for the operative strategy. The first and most frequently explored clinical application was resection and reconstruction of oro-mandibular tumours due to their easier medical image processing in comparison with other fields. The development of 3DP operative templates for cutting and/ or reconstruction guides minimised the surgeon's fatigue and complication rates, and optimised the operating room time, which led to lower morbidity. Similar approaches have been employed for complex cases of temporal bone and sinonasal surgery.

Clinical benefits were advocated by the authors to justify the main limitations of AM technology: costs, necessity for technical skills and technological availability. Costeffectiveness was widely debated in literature: the decreased surgical time and employment of self-fabricated 3DP models or guides (instead of outsourced manufacturing) appeared to counter balance the price of the starting technological investments and the technical skills required 
for pre- and postprocessing printing activity ${ }^{94}$. Interestingly, for $34 \%$ of studies on customised surgical planning, a specific description of the technology adopted was not available (Fig. 4): this arises from the choice of externalization of the 3D printing process, as often declared by authors themselves 45778093110121 . To date, the rapid expansion of AM machines and materials has significantly lowered costs, making this technology more accessible. The most employed technology in this field of application was power bed fusion $(27 \%)$, which offers medical grade materials (like titanium, or biocompatible polyamide) to be used as intra-operative templates, followed by material extrusion (12\%), which also offers biocompatible materials, even if with lower printing resolution. Surgical and preclinical education represents the second most studied 3DP application. Surgical training traditionally made use of physical models, animals, or human cadavers. The adoption of both fixed and fresh human specimens in labs has long been and still is a core component in training for ENT surgery, but it has certain limitations such as transmission of infectious agents, exposure to potentially carcinogenic formaldehyde and excessive costs. More recently, 3DP models were used in the teaching of complex anatomy and to simulate critical surgical procedures with particular regard to temporal bone and skull base dissection. The most employed AM technology for this application (Fig. 4) was material extrusion (39\%): this is not surprising, since this is the most affordable technology, especially in terms of printing materials. Material extrusion is actually the most suited to apply for teaching and training, where models are usually subjected to damage and need to be produced in high numbers. $25 \%$ of studies used power bed fusion machines, thanks to the availability of materials (e.g. polyamide) with mechanical properties that are suitable for drilling and dissection operations. The complexity of temporal bone anatomy and related surgical procedures, essentially based on bone drilling and removal, explain the extensive research on this issue.

The evolution of 3DP systems and materials has enabled the reproduction of even the finest chromatic details and mechanical properties of the object resulting in highly representative 3DP simulators. These solutions are unfortunately still expensive, and consequently less employed for the production of didactic devices, as confirmed by the limited use of technologies with high chromatic resolution (binder jetting, 11\%) and with tuneable mechanical properties (material jetting, 11\%).

Tissue engineering and implantable prostheses is discussed in fewer reports since it represents the most recent 3DP application, but it also entails more exciting future perspectives. The current literature reported the applica- tion of 3DP customised titanium alloy prostheses in 33 cases of mandibular reconstruction after tumour resection. Power bed fusion is confirmed as the most widely employed technology in the field, used in 50\% of studies: the most common materials are titanium and cobalt-chrome, which are also widely employed in implant standard manufacturing. Preliminary data have provided encouraging results in terms of safety and effectiveness, opening new frontiers of investigation.

Nowadays, AM technology has been involved in the production of biocompatible matrices aimed to be cellularised (scaffold), hence forming a new functional tissue. ENT scaffold research is at present confined to a preclinical stage (in vitro and animal testing), with relevant applications in the reconstruction of the upper aerodigestive tract ${ }^{133134}$, replacement of tympanic membrane ${ }^{135}$ and plastic rebuilding of auricular and nasal cartilages ${ }^{136} 137$. Even though scaffold research is in its infancy, it represents a future direction of high interest. New perspectives will concern the microstructure of 3DP scaffolds to overcome many currently unsolved questions as well as proper vascularisation to avoid cell degeneration and adequate stem cell proliferation/specialisation. The final goal would entail functional aspects to produce functional tissues and organs by involvement of multiple types of cells and biomaterials.

Moreover, in the foreseeable future, technical advancements will possibly provide a better solution to issues involving biocompatibility and sterilisation protocols of 3DP materials.

\section{Conclusions}

3DP systems have revolutionised prototyping in the industrial field by lowering production time from days to hours and costs from thousands to only a few dollars. Today, 3D printers are no longer confined to prototyping, but are increasingly employed in the medical discipline with fascinating results, even in many aspects of otorhinolaryngology. Nevertheless, current reports are still limited to small case-series of patients and lack of comparative objective data to validate 3DP technology in daily clinical practice. 3DP bioengineering is at the beginning of an exciting research field, and the positive results to date are far from what it will be possible to achieve in forthcoming clinical applications.

\section{References}

1 Kodama H. Automatic method for fabricating a three-dimensional plastic model with photo-hardening polymer. Rev Sci Instrum 1981;52:1770-3. 
2 Herbert A. Solid object generation. J Appl Photogr Eng 1982;8:185-8.

3 Hull C. Apparatus for production of three-dimensional object by stereolithography. U.S. PATENT US4575330, 1986. doi:10.1145/634067.634234.

4 Schubert C, Van Langeveld M, Donoso L. Innovations in $3 D$ printing : a $3 D$ overview from optics to organs. $\mathrm{Br} \mathrm{J}$ Ophtalmol 2014;98:159-61.

5 NASA to launch $3 D$ printer into space to help supply astronauts. Theregister.coUK. 2013;October 1.

6 Banks J. Adding value in additive manufacturing: researchers in the United Kingdom and Europe look to 3D printing for customization. IEEE Pulse 2013;4:22-6.

7 Canzi P, Marconi S, Manfrin M, et al. From CT scanning to $3 D$ printing technology: a new method for the preoperative planning of a transcutaneous bone-conduction hearing device. Acta Otorhinolaryngol Ital 2018;38:251-6.

8 Auricchio F, Marconi S. 3D printing: clinical applications in orthopaedics and traumatology. EFORT Open Rev 2017;1:121-7.

9 Gibson I, Rosen D, Stucker B. Additive manufacturing technologies: $3 d$ printing, rapid prototyping, and direct digital manufacturing. $2^{\text {nd }}$ Edition. Johnson Matthey Technol Rev 2015;59:1993-8.

10 No authors listed. Committee F42 on additive manufacturing technologies.http:// www.astm.org/COMMITTEE/ F42.htm.

11 Moher D, Liberati A, Tetzlaff, et al. Preferred reporting items for systematic reviews and meta-analyses: the PRISMA Statement. J Clin Epidemiol 2009;62:1006-12.

12 Cohen J, Reyes S. Creation of a $3 D$ printed temporal bone model from clinical CT data. Am J Otolaryngol 2015;36:619-24.

13 Da Cruz M, Francis H. Face and content validation of a novel three-dimensional printed temporal bone for surgical skills development. J Laryngol Otol 2015;129:23-29.

14 Hochman J, Rhodes C, Kraut J, et al. End user comparison of anatomically matched 3-Dimensional printed and virtual haptic temporal bone simulation: a pilot study. Otolaryngol Head Neck Surg 2015;153:263-8.

15 Hochman J, Rhodes C, Wong D, et al. Comparison of cadaveric and isomorphic three-dimensional printed models in temporal bone education. Laryngoscope 2015;125:2353-7.

16 Longfield E, Brickman T, Jeyakumar A. 3D printed pediatric temporal bone: a novel training model. Otol Neurotol 2015;36:793-5.

17 Mowry S, Jammal H, Myer C $4^{\text {th }}$, et al. A novel temporal bone simulation model using $3 D$ printing techniques. Otol Neurotol 2015;36:1562-5.

18 Rose A, Kimbell J, Webster C, et al. Multi-material 3D models for temporal bone surgical simulation. Ann Otol Rhinol Laryngol 2015;124:528-36.

19 Hochman J, Kraut J, Kazmerik K, et al. Generation of a
$3 D$ printed temporal bone model with internal fidelity and validation of the mechanical construct. Otolaryngol Head Neck Surg 2014;150:448-54.

20 Unger B, Kraut J, Rhodes C, et al. Design and validation of $3 D$ printed complex bone model with internal anatomic fidelity for surgical training and rehearsal. Stud Heal Technol Inform 2014;196:439-45.

21 Mick P, Arnoldner C, Mainprize J, et al. Face validity study of an artificial temporal bone for simulation surgery. Otol Neurotol 2013;34:1305-10.

22 Roosli C, Sim J, Möckel H, et al. An artificial temporal bone as a training tool for cochlear implantation. Otol Neurotol 2013;34:1048-51.

23 Bakhos D, Velut S, Robier A, et al. Three-Dimensional modeling of the temporal bone for surgical training. Otol Neurotol 2010;31:328-34.

24 Mori K. Dissectable modified three-dimensional temporal bone and whole skull base models for training in skull base approaches. Skull Base 2009;19:333-43.

25 Mori K, Yamamoto T, Oyama K, et al. Modification of three-dimensional prototype temporal bone model for training in skull-base surgery. Neurosurg Rev 2009;32:233-9.

26 Mori K, Yamamoto T, Oyama K, et al. Modified three-dimensional skull base model with artificial dura mater, cranial nerves, and venous sinuses for training in skull base surgery: technical note. Neurol Med Chir 2008;48:582-7.

27 Suzuki M, Hagiwara A, Ogawa Y, et al. Rapid-prototyped temporal bone and inner-ear models replicated by adjusting computed tomography thresholds. J Laryngol Otol 2007;121:1025-8.

${ }^{28}$ Grunert R, Strauss G, Moeckel H, et al. ElePhant - an anatomical electronic phantom as simulation-system for otologic surgery. Conf Proc IEEE Eng Med Biol Soc 2006;1:4408-11.

29 Suzuki M, Ogawa Y, Kawano A, et al. Rapid prototyping of temporal bone for surgical training and medical education. Acta Otolaryngol 2004;124:400-2.

30 Suzuki M, Ogawa Y, Hagiwara A, et al. Rapidly prototyped temporal bone model for otological education. ORL J Otorhinolaryngol Relat Spec 2004;66:62-4.

31 Begall K, Vorwerk U. Artificial petrous bone produced by stereolithography for microsurgical dissecting exercises. ORL J Otorhinolaryngol Relat Spec 1998;60:241-5.

32 Monfared A, Mitteramskogler G, Gruber S, et al. High-fidelity, inexpensive surgical middle ear simulator. Otol Neurotol 2012;33:1573-7.

33 Barber S, Kozin E, Dedmon M, et al. 3D-printed pediatric endoscopic ear surgery simulator for surgical training. Int J Pediatr Otorhinolaryngol 2016;90:113-8.

34 Kuru I, Maier H, Müller M, et al. A 3D-printed functioning anatomical human middle ear model. Hear Res 2016;340:204-13.

35 Rose A, Webster C, Harrysson OL, et al. Pre-operative 
simulation of pediatric mastoid surgery with 3D-printed temporal bone models. Int J Pediatr Otorhinolaryngol 2015;79:740-4.

36 Suzuki M, Hagiwara A, Kawaguchi S, et al. Application of a rapid-prototyped temporal bone model for surgical planning. Acta Otolaryngol 2005;125:29-32.

37 Löppönen H, Holma T, Sorri M, et al. Computed tomography data based rapid prototyping model of the temporal bone before cochlear implant surgery. Acta Otolaryngol Suppl 1997;529:47-9.

38 Andrews J, Mankovich N, Anzai Y, et al. Stereolithographic model construction from CT for assessment and surgical planning in congenital aural atresia. Am J Otol 1994;15:335-9.

39 Pai I, Rojas P, Jiang D, et al. The use of $3 D$ printed external and internal templates for bonebridge implantation: technical note. Clin Otolaryngol 2017;42:1118-20.

40 Matsumoto N, Takumi Y, Cho B, et al. Template-guided implantation of the Bonebridge: clinical experience. Eur Arch Otorhinolaryngol 2015;272:3669-75.

${ }^{41}$ Cho B, Matsumoto N, Mori M, et al. Image-guided placement of the Bonebridge without surgical navigation equipment. Int J Comput Assist Radiol Surg 2014;9:845-55.

42 Takumi Y, Matsumoto N, Cho B, et al. A clinical experience of "STAMP" plate-guided Bonebridge implantation. Acta Otolaryngol 2014;134:1042-6.

43 Yamashita M, Matsumoto N, Cho B, et al. Registration using 3D-printed rigid templates outperforms manually scanned surface matching in image-guided temporal bone surgery. Int J Comput Assist Radiol Surg 2016;11:211927.

44 Ritacco L, Di Lella F, Mancino A, et al. 3D printed models and navigation for skull base surgery: case report and virtual validation. Stud Heal Technol Inform 2015;216:1025.

45 Oka M, Cho B, Matsumoto N, et al. A preregistered STAMP method for image-guided temporal bone surgery. Int J Comput Assist Radiol Surg 2014;9:119-26.

46 Cho B, Oka M, Matsumoto N, et al. Warning navigation system using real-time safe region monitoring for otologic surgery. Int J Comput Assist Radiol Surg 2013;8:395-405.

47 Matsumoto N, Oka M, Cho B, et al. Cochlear implantation assisted by noninvasive image guidance. Otol Neurotol 2012;33:1333-8.

48 Matsumoto N, Hong J, Hashizume M, et al. A minimally invasive registration method using surface template-assisted marker positioning (STAMP) for image-guided otologic surgery. Otolaryngol Head Neck Surg 2009;140:96-102.

49 Muelleman T, Peterson J, Chowdhury N, et al. Individualized surgical approach planning for petroclival tumors using a $3 D$ printer. J Neurol Surg B Skull Base 2016;77:243-8.

50. Kozin E, Remenschneider A, Cheng S, et al. Three-dimensional printed prosthesis for repair of superior canal dehiscence. Otolaryngol Head Neck Surg 2015;153:616-9.
51. Chang D, Lin R, Bowe S, et al. Fabrication and validation of a low-cost, medium-fidelity silicone injection molded endoscopic sinus surgery simulation model. Laryngoscope 2017;127:781-6.

52 Tai B, Wang A, Joseph J, et al. A physical simulator for endoscopic endonasal drilling techniques: technical note. J Neurosurg 2016;124:811-6.

53 Narayanan V, Narayanan P, Rajagopalan R, et al. Endoscopic skull base training using $3 D$ printed models with pre-existing pathology. Eur Arch Otorhinolaryngol 2015;272:753-7.

54 Chan H, Siewerdsen J, Vescan A, et al. 3D rapid prototyping for otolaryngology-head and neck surgery: applications in image-guidance, surgical simulation and patient-specific modeling. PLoS One 2015;10:1-18.

55 AlReefi M, Nguyen L, Mongeau L, et al. Development and validation of a septoplasty training model using 3-dimensional printing technology. Int Forum Allergy Rhinol 2017;7:399-404.

56 Chiesa Estomba C, González Fernández I, Iglesias Otero MA. How we do it: anterior and posterior nosebleed trainer, the $3 D$ printing epistaxis project. Clin Otolaryngol 2016:25:1-2.

57 Sander I, Mcgoldrick M, Helms M, et al. Three-dimensional printing of X-ray computed tomography datasets with multiple materials using open-source data processing. Anat Sci Educ 2017;10:383-91.

58 Onerci Altunay Z, Bly J, Edwards P, et al. Three-dimensional printing of large nasal septal perforations for optimal prosthetic closure. Am J Rhinol Allergy 2016;30:287-93.

59 Daniel M, Watson J, Hoskison E, et al. Frontal sinus models and onlay templates in osteoplastic flap surgery. J Laryngol Otol 2011;125:82-5.

60 Raos P, Klapan I, Galeta T. Additive manufacturing of medical models - applications in rhinology. Coll Antropol 2015;39:667-73.

61 Nahumi N, Shohet M, Bederson J, et al. Frontorbital fibrous dysplasia resection and reconstruction with custom polyetherlatone alloplast. J Craniofac Surg 2015;26:720-2.

62. Kavanagh K, Cote V, Tsui Y, et al. Pediatric laryngeal simulator using $3 D$ printed models: a novel technique. Laryngoscope 2017;127:132-7.

63 Johnson C, Howell J, Mettenburg D, et al. Mechanical modeling of the human cricoid cartilage using computer-aided design: applications in airway balloon dilation research. Ann Otol Rhinol Laryngol 2016;125:69-76.

64 Ainsworth T, Kobler J, Loan G, et al. Simulation model for transcervical laryngeal injection providing real-time feedback. Ann Otol Rhinol Laryngol 2014;123:881-6.

65 Govsa F, Yagdi T, Ozer M, et al. Building 3D anatomical model of coiling of the internal carotid artery derived from CT angiographic data. Eur Arch Otorhinolaryngol 2017;274:1097-102.

66 Grolman W, Schouwenburg P, Verbeeten $\mathrm{Bj}$, et al. Three-dimensional models of the tracheostoma using 
stereolithography. ORL J Otorhinolaryngol Relat Spec 1995;57:338-42.

67 Bosc R, Hersant B, Carloni R, et al. Mandibular reconstruction after cancer: an in-house approach to manufacturing cutting guides. Int J Oral Maxillofac Surg 2017;46:24-31.

68 Rachmiel A, Shilo D, Blanc O, et al. Reconstruction of complex mandibular defects using integrated dental custom-made titanium implants. Br J Oral Maxillofac Surg 2017;55:425-7.

69 Shah S, O'Connor R, Watson J, et al. Use of three-dimensional printing to assess transport vectors in mandibular distraction osteogenesis. Br J Oral Maxillofac Surg 2017;55:74-6.

70 Lee U, Kwon J, Woo S, et al. Simultaneous bimaxillary surgery and mandibular reconstruction with a 3-dimensional printed titanium implant fabricated by Electron Beam Melting: a preliminary mechanical testing of the printed mandible. J Oral Maxillofac Surg 2016;74:1501-15.

${ }^{71}$ Lim S, Kim M, Kang S. Precision of fibula positioning guide in mandibular reconstruction with a fibula graft. Head Face Med 2016;12:1-10.

72 Numajiri T, Nakamura H, Sowa Y, et al. Low-cost design and manufacturing of surgical guides for mandibular reconstruction using a fibula. Plast Reconstr Surg Glob Open 2016;4:1-9.

73 Yamada H, Nakaoka K, Sonoyama T, et al. Clinical usefulness of mandibular reconstruction using custom-made titanium mesh tray and autogenous particulate cancellous bone and marrow harvested from tibia and/or Ilia. J Craniofac Surg 2016;27:586-92.

74 Man Q, Jia J, Liu K, et al. Secondary reconstruction for mandibular osteoradionecrosis defect with fibula osteomyocutaneous flap flowthrough from radial forearm flap using stereolithographic 3-dimensional printing modeling technology. J Craniofac Surg 2015;26:190-3.

75 Modabber A, Möhlhenrich S, Ayoub N, et al. Computer-aided mandibular reconstruction with vascularized iliac crest bone flap and simultaneous implant surgery. $\mathrm{J}$ Oral Implant 2015;41:189-94.

76 Reiser V, Alterman M, Shuster A, et al. V-stand-a versatile surgical platform for oromandibular reconstruction using a 3-dimensional virtual modeling system. J Oral Maxillofac Surg 2015;73:1211-26.

77 Schepers R, Raghoebar G, Vissink A, et al. Accuracy of fibula reconstruction using patient-specific CAD/CAM reconstruction plates and dental implants: A new modality for functional reconstruction of mandibular defects. $\mathrm{J}$ Craniomaxillofac Surg 2015;43:649-57.

78 Shan X, Chen H, Liang J, et al. Surgical reconstruction of maxillary and mandibular defects using a printed titanium mesh. J Oral Maxillofac Surg 2015;73:1437.e1-9.

79 Steinbacher D. Three-Dimensional analysis and surgical planning in craniomaxillofacial surgery. J Oral Maxillofac Surg. 2015;73:40-56.
80 Succo G, Berrone M, Battiston B, et al. Step-by-step surgical technique for mandibular reconstruction with fibular free flap: application of digital technology in virtual surgical planning. Eur Arch Otorhinolaryngol 2015;272:1491-501.

81 Wilde F, Hanken H, Probst F, et al. Multicenter study on the use of patient-specific CAD/CAM reconstruction plates for mandibular reconstruction. Int J CARS 2015;10:2035-51.

82 Ayoub N, Ghassemi A, Rana M, et al. Evaluation of computer-assisted mandibular reconstruction with vascularized iliac crest bone graft compared to conventional surgery: a randomized prospective clinical trial. Trials 2014;15:1-13.

83 Azuma M, Yanagawa T, Ishibashi-Kanno N, et al. Mandibular reconstruction using plates prebent to fit rapid prototyping 3-dimensional printing models ameliorates contour deformity. Head Face Med 2014;10:1-8.

84 de Farias T, Dias F, Galvão M, et al. Use of prototyping in preoperative planning for patients with head and neck tumors. Head Neck 2014;36:1773-82.

85 Liu Y, Xu L, Zhu H, et al. Technical procedures for template-guided surgery for mandibular reconstruction based on digital design and manufacturing. Biomed Eng Online 2014;13:1-15.

86 Modabber A, Ayoub N, Möhlhenrich S, et al. The accuracy of computer-assisted primary mandibular reconstruction with vascularized bone flaps: iliac crest bone flap versus osteomyocutaneous fibula flap. Med Devices (Auckl) 2014;16:211-7.

87 Tsai M, Wu C. Study of mandible reconstruction using a fibula flap with application of additive manufacturing technology. Biomed Eng Online 2014;13:1-15.

88 Watson J, Hatamleh M, Alwahadni A, et al. Correction of facial and mandibular asymmetry using a computer aided design/computer aided manufacturing prefabricated titanium implant. J Craniofac Surg 2014;25:1099-101.

89 Wilde F, Cornelius C, Schramm A. Computer-assisted mandibular reconstruction using a patient-specific reconstruction plate fabricated with computer-aided design and manufacturing techniques. Craniomaxillofac Trauma Reconstr 2014;7:158-66.

90 Yamada H, Nakaoka K, Horiuchi T, et al. Mandibular reconstruction using custom-made titanium mesh tray and particulate cancellous bone and marrow harvested from bilateral posterior ilia. J Plast Surg Hand Surg 2014;48:183-90.

91 Coppen C, Weijs W, Bergé S, et al. Oromandibular reconstruction using $3 D$ planned triple template method. J Oral Maxillofac Surg 2013;71:243-7.

92 Foley B, Thayer W, Honeybrook A, et al. Mandibular reconstruction using computer-aided design and computer-aided manufacturing: an analysis of surgical results. $\mathrm{J}$ Oral Maxillofac Surg 2013;71:111-9.

93 Hanasono M, Skoracki R. Computer-assisted design and 
rapid prototype modeling in microvascular mandible reconstruction. Laryngoscope 2013;123:597-604.

94 Mazzoni S, Marchetti C, Sgarzani R, et al. Prosthetically guided maxillofacial surgery: evaluation of the accuracy of a surgical guide and custom-made bone plate in oncology patients after mandibular reconstruction. Plast Reconstr Surg 2013;131:1376-85.

95 Zheng G, Su Y, Liao G, et al. Mandibular reconstruction assisted by preoperative simulation and accurate transferring templates : preliminary report of clinical application. J Oral Maxillofac Surg 2013;71:1613-8.

96 Ciocca L, Mazzoni S, Fantini M, et al. CAD/CAM guided secondary mandibular reconstruction of a discontinuity defect after ablative cancer surgery. J Craniomaxillofac Surg 2012;40:511-5.

97 Ciocca L, Mazzoni S, Fantini M, et al. The design and rapid prototyping of surgical guides and bone plates to support iliac free flaps for mandible reconstruction. Plast Reconstr Surg 2012;129:859-61.

98 Dérand P, Rännar L, Hirsch J. Imaging, virtual planning, design, and production of patient-specific implants and clinical validation in craniomaxillofacial surgery. Craniomaxillofac Trauma Reconstr 2012;5:137-44.

99 Hou J, Chen M, Pan C, et al. Application of CAD / CAM-assisted technique with surgical treatment in reconstruction of the mandible. J Craniomaxillofac Surg 2012;40:432-7.

${ }^{100}$ Lethaus B, Poort L, Böckmann R, et al. Additive manufacturing for microvascular reconstruction of the mandible in 20 patients. J Craniofac Surg 2012;40:43-6.

${ }^{101}$ Modabber A, Legros C, Rana M, et al. Evaluation of computer-assisted jaw reconstruction with free vascularized fibular flap compared to conventional surgery: a clinical pilot study. Int J Med Robot 2012;8:215-20.

${ }^{102}$ Modabber A, Gerressen M, Stiller M, et al. Computer-assisted mandibular reconstruction with vascularized iliac crest bone graft. Aesthetic Plast Surg 2012;36:653-9.

${ }^{103}$ Patel A, Levine J, Brecht L, et al. Digital technologies in mandibular pathology and reconstruction. Atlas Oral Maxillofac Surg Clin North Am 2012;20:95-106.

${ }^{104}$ Sink J, Hamlar D, Kademani D, et al. Computer-aided stereolithography for presurgical planning in fibula free tissue reconstruction of the mandible. $\mathrm{J}$ Reconstr Microsurg 2012;28:395-403.

105 Wilde F, Plail M, Riese C, et al. Mandible reconstruction with patient-specific pre-bent reconstruction plates: comparison of a transfer key method to the standard methodresults of an in vitro study. Int $\mathrm{J}$ Comput Assist Radiol Surg 2012;7:57-63.

${ }^{106}$ Zheng G, Su Y, Liao G, et al. Mandible reconstruction assisted by preoperative simulation and transferring templates: cadaveric study of accuracy. J Oral Maxillofac Surg 2012;70:1480-5.

${ }^{107}$ Abou-Elfetouh A, Barakat A, Abdel-Ghany K. Computerguided rapid-prototyped templates for segmental mandib- ular osteotomies: a preliminary report. Int J Med Robot Comput Assist Surg 2011;7:187-92.

${ }^{108}$ Antony A, Chen W, Kolokythas A, et al. Use of virtual surgery and stereolithography-guided osteotomy for mandibular reconstruction with the free fibula. Plast Reconstr Surg 2011;128:1080-4.

${ }^{109}$ Bell R, Weimer K, Dierks E, et al. Computer planning and intraoperative navigation for palatomaxillary and mandibular reconstruction with fibular free flaps. J Oral Maxillofac Surg 2011;69:724-32.

${ }^{110}$ Hou J, Chen M, Pan C, et al. Immediate reconstruction of bilateral mandible defects: management based on computer-aided design/computer-aided manufacturing rapid prototyping technology in combination with vascularized fibular osteomyocutaneous flap. J Oral Maxillofac Surg 2011;69:1792-7.

${ }^{111}$ Mehra P, Miner J, D'Innocenzo R, et al. Use of 3-D stereolithographic models in oral and maxillofacial surgery. J Maxillofac Oral Surg 2011;10:6-13.

${ }^{112}$ Yamanaka Y, Yajima H, Kirita T, et al. Mandibular reconstruction with vascularised fibular osteocutaneous flaps using prefabricated stereolithographic mandibular model. J Plast Reconstr Aesthet Surg 2010;63:1751-3.

113 Zhou L, Shang H, He L, et al. Accurate reconstruction of discontinuous mandible using a reverse engineering/ computer-aided design/rapid prototyping technique: a preliminary clinical study. J Oral Maxillofac Surg 2010;68:2115-21.

${ }^{114}$ Cohen A, Laviv A, Berman P, et al. Mandibular reconstruction using stereolithographic 3-dimensional printing modeling technology. Oral Surg Oral Med Oral Pathol Oral Radiol Endod 2009;108:661-6.

${ }^{115}$ Fariña R, Plaza C, Martinovic G. New transference technique of position of mandibular reconstructing plates using stereolithographic models. J Oral Maxillofac Surg 2009;67:2544-8.

116 Juergens P, Krol Z, Zeilhofer H, et al. Computer simulation and rapid prototyping for the reconstruction of the mandible. J Oral Maxillofac Surg 2009;67:2167-70.

${ }^{117}$ Leiggener C, Messo E, Thor A, et al. A selective laser sintering guide for transferring a virtual plan to real time surgery in composite mandibular reconstruction with free fibula osseous flaps. Int J Oral Maxillofac Surg 2009;38:187-92.

${ }^{118}$ Liu X, Gui L, Mao C, et al. Applying computer techniques in maxillofacial reconstruction using a fibula flap: a messenger and an evaluation method. J Craniofac Surg 2009;20:372-377.

119. Chow L, Cheung L. The usefulness of stereomodels in maxillofacial surgical management. J Oral Maxillofac Surg 2007;65:2260-8.

${ }^{120}$ Lee J, Fang J, Chang L, et al. Mandibular defect reconstruction with the help of mirror imaging coupled with laser stereolithographic modeling technique. J Formos Med Assoc 2007;106:244-50. 
${ }^{121}$ Ro E, Ridge J, Topham N. Using stereolithographic models to plan mandibular reconstruction for advanced oral cavity cancer. Laryngoscope 2007;117:759-61.

122 Toro C, Robiony M, Costa F, et al. Feasibility of preoperative planning using anatomical facsimile models for mandibular reconstruction. Head Face Med 2007;3:1-11.

${ }^{123}$ Yeung R, Samman N, Cheung L, et al. Stereomodel-assisted fibula flap harvest and mandibular reconstruction. J Oral Maxillofac Surg 2007;65:1128-134.

${ }^{124}$ Hallermann W, Olsen S, Bardyn T, et al. A new method for computer-aided operation planning for extensive mandibular reconstruction. Plast Reconstr Surg 2006;117:24317.

${ }^{125}$ Hannen E. Recreating the original contour in tumor deformed mandibles for plate adapting. Int J Oral Maxillofac Surg 2006;35:183-5.

${ }^{126}$ Cunningham L, Madsen M, Peterson G. Stereolithographic modeling technology applied to tumor resection. J Oral Maxillofac Surg 2005;63:873-8.

${ }^{127}$ Wong T, Fang J, Chung C, et al. Comparison of 2 methods of making surgical models for correction of facial asymmetry. J Oral Maxillofac Surg 2005;63:200-8.

${ }^{128}$ Singare S, Dichen L, Bingheng L, et al. Design and fabrication of custom mandible titanium tray based on rapid prototyping. Med Eng Phys 2004;26:671-6.

${ }^{129}$ Kernan B, Wimsatt J 3rd. Use of a stereolithography model for accurate, preoperative adaptation of a reconstruction plate. J Oral Maxillofac Surg 2000;58:349-51.
${ }^{130}$ Komori T, Takato T, Akagawa T. Use of a laser-hardened three-dimensional replica for simulated surgery. J Oral Maxillofac Surg 1994;52:516-21.

${ }^{131}$ Wiedermann J, Joshi A, Jamshidi A, et al. Utilization of a submental island flap and $3 D$ printed model for skull base reconstruction: infantile giant cranio-cervicofacial teratoma. Int J Pediatr Otorhinolaryngol 2017;92:143-5.

${ }^{132}$ Paydarfar J, Wu X, Halter R. MRI-and CT-compatible polymer laryngoscope: a step toward image-guided transoral surgery. Otolaryngol Head Neck Surg 2016;155:364-6.

${ }^{133}$ Dorati R, De Trizio A, Marconi S, et al. Design of a bioabsorbable multilayered patch for esophagus tissue engineering. Macromol Biosci 2017;17:1-11.

${ }^{134}$ Zopf D, Flanagan C, Wheeler M, et al. Treatment of severe porcine tracheomalacia with a 3-dimensionally printed, bioresorbable, external airway splint. JAMA Otolaryngol Head Neck Surg 2014;140:66-71.

${ }^{135}$ Mota C, Danti S, D'Alessandro D, et al. Multiscale fabrication of biomimetic scaffolds for tympanic membrane tissue engineering. Biofabrication 2015;7:1-22.

${ }^{136}$ Zopf D, Mitsak A, Flanagan C, et al. Computer-aided designed, 3-dimensionally printed porous tissue bioscaffolds for craniofacial soft tissue reconstruction. Otolaryngol Head Neck Surg 2015;152:57-62.

${ }^{137}$ Kang H, Lee S, Ko I, et al. A 3D bioprinting system to produce human-scale tissue constructs with structural integrity. Nat Biotechnol 2016;34:312-9.

Received: October 30, 2017- Accepted: May 14, 2018

Address for correspondence: Pietro Canzi, University of Pavia, Department of Otorhinolaryngology, IRCCS Policlinico San Matteo Foundation, viale Camillo Golgi 19, 27100, Pavia, Italy. Fax +39 0382 528184. E-mail: pietro.canzi@unipv.it. 